This article has been scanned by iThenticat No plagiarism detected

Volume 3, Issue 6, December 2021

p. $315-322$

\title{
EXPLORING THE IMPORTANCE OF PLURILINGUAL COMPETENCE IN THE PRE- SERVICE TEACHER TRAINING: A CASE STUDY OF TEACHER TRAINING COLLEGES OF ALGERIA
}

http://dx.doi.org/10.47832/2757-5403.6-3.22

\section{Souhila BENZERROUG ${ }^{1}$ \& Samah BENZERROUG ${ }^{2}$}

\begin{abstract}
:
The present research paper highlights the importance of plurilingual competence to language education in pre-service teacher training at the Teacher Training College of Bouzareah-Algeria-. The study is designed to gain insight into the development of pluringual competence in the pre-service program that is addressed to the students of the departments of French and English. It aims at enhancing the teaching and learning of foreign languages in order to meet the universal requirements related to interculturality and plurilingualism.To achieve the above mentioned aims, the researchers interviewed ENSB teacher trainers to investigate their perceptions towards the teaching of that competence. A qualitative method was then employed by using a semistructured interview with university teachers of Didactics and Language Studies in order to identify the extent of interest that is assigned to the development of plurilingual competence in the teaching practices as well as the syllabus content.
\end{abstract} Received: $15 / 10 / 2021$

Key words: Professionalizing Training, Plurilingualism, Foreign Languages, Perceptions.

\footnotetext{
${ }^{1}$ Dr., Teacher Training College (ENSB), Algeria, houd-bens@live.fr, https://orcid.org/0000-0002-1550-2770

${ }^{2}$ Dr., Teacher Training College (ENSB), Algeria, benz-sam@hotmail.fr, https://orcid.org/0000-0002- 5543-1110
}

Copyright $(\odot$ Published by IJHER Journal, www.ijherjournal.com Rimar Academy, Fatih, Istanbul, 34093 Turkey

All rights reserved 


\section{PLACE DE LA COMPETENCE PLURILINGUE DANS LA FORMATION DES FORMATEURS: CAS DES ENS D'ALGERIE}

\section{Souhila BENZERROUG Samah BENZERROUG}

\section{Résumé}

Cet article aborde la thématique de la compétence plurilingue dans la formation des futurs enseignants de langues au sein de l'École Normale Supérieure de Bouzaréah en Algérie. Nous nous proposons de démontrer sa place dans les programmes de formation dans les deux disciplines, à savoir la langue française et la langue anglaise. Notre but étant de promouvoir l'enseignement des langues afin de pallier aux nouvelles exigences mondiales liées à l'interculturalité et au plurilinguisme. Nous tenterons ainsi d'interroger les formateurs des formateurs sur leurs représentations à propos de l'enseignement de cette compétence. Pour ce faire, nous avons tenté d'interroger les enseignants universitaires des deux disciplines: sciences du langage et didactique de 1'ENS-B à travers un entretien semi-directif à propos de la place de la compétence plurilingue dans leurs pratiques de classe et les contenus de la formation qu'ils assurent.

Mots clés: Formation Professionnalisante, Plurilinguisme, Langues Étrangères, Représentations.

\section{1-Introduction}

La formation des formateurs a toujours préoccupé les chercheurs que ce soit en didactique des langues, en éducation ou alors en didactique professionnelle. En effet, , celle -ci est la base de tout processus enseignement-apprentissage et ce pour assurer "une formation professionnelle au métier d'enseignant " comme le soulignent (Gruca, 2017, pp. 136-137). Les recherches actuelles sont accès beaucoup plus sur la didactique du plurilinguisme et l'interculturalité compte tenu de leur dominance dans la vie de tous les jours et au sein de la société et c'est l'objectif primordial pour le CECRL: " Le plurilinguisme de tout citoyen est l'un des objectifs principaux de la politique linguistique du conseil de l'Europe. " (Cuq, 2003, p. 196)

Beaucoup de chercheurs ont abordé le plurilinguisme (Causa, (2012)) traite la thématique de la Réflexions autour de la mise en place d'une éducation au(x) plurilinguisme(s) en formation initiale à l'enseignement des langues. Elle propose qu'il y ait un travail important reste à accomplir. Ce travail devra alors se penser davantage sur la formation initiale dans une perspective collaborative. Une autre recherche de Martine Derivry-Plard (Derivry-Plard, 2015) s'interroge sur la reconceptualisation de la formation des enseignants selon un paradigme plurilingue et pluriculturel. Le travail d'Estela Klett (Klet, 2017) nous propose de jumeler à la fois les trois concepts suivants: l'internationalisation, le plurilinguisme et la formation des enseignants. La chercheure nous explique que: "On peut donc imaginer qu'il faut enrichir le capital linguistique pour être à la hauteur des circonstances, la connaissance de l'anglais étant nécessaire mais pas suffisante. C'est à ce moment là qu'interviendrait le formateur. " (Klet, 2017, p. 68)

Notre travail est accès dans cet article sur l'articulation entre la formation et leplurilinguisme. Nous tentons en tant qu'enseignantes en didactique des langues dans les deux départements de français et d'anglais de voir la place qu'occupe la compétence plurilingue dans la formation initiale des futurs enseignants à l'ENS de Bouzaréah. 
Notre question principale serait: est ce que les formateurs des formateurs sont conscients de l'importance de la compétence plurilingue et comment la prennent -ils en charge dans le contexte universitaire pour une formation professionnalisante?

D'autres questions articulent notre travail de recherche: les enseignants formateurs de l'ENS de Bouzaréah, ont-ils été formés durant leur formation initiale au plurilinguisme? Maitrisent -ils le concept du plurilinguisme ? Comment perçoivent-ils l'usage des différentes langues en classe ? Préparent -ils les futurs formés sur le rôle de la compétence plurilingue en classe de FLE ? Est-ce les programmes dédiés à la formation en didactique et en sciences du langage prennent -ils en considération cette dimension ? Ya t-i une concordance entre ces programmes et la réalité du terrain? Quelles sont les recommandations pour bonne prise en charge de la pluralité dans la formation des futurs enseignants ?

Cette recherche s'inscrit donc en pédagogie universitaire et en didactique du plurilinguisme à la fois car nous allons nous intéresser l'enseignement de la compétence plurilingue dans le contexte universitaire.

Nous allons structurer notre article comme suit: dans un premier cas nous allons nous intéresser à la formation des formateurs à l'ENS-B3 et à la formation professionnalisante. Dans un second cas, nous tenterons de décrire les programmes dédiés à la compétence plurilingue dans les deux spécialités cités supra. Nous présentons enfin les outils d'investigation et les résultats du dépouillement du corpus recueilli. Des recommandations et des perspectives seront émises à la fin pour une bonne prise en charge de la compétence plurilingue dans la formation des formateurs.

\section{2-La formation professionnalisante des formateurs}

La formation des formateurs occupe une place prépondérante dans le monde entier et c'est "l'une des caractéristiques du champ du FLES, (.....), à savoir une formation continue à la formation initiale " (Cuq, 2003, p. 133)

La compétence professionnelle d'un enseignant est alors composée d'un certain nombre d'éléments décrits couramment sous la forme d'un profil. (Cuq, 2003, p. 104)

Il est existe deux types de formation: la formation initiale et continue. Toutefois et en ce qui concerne les savoirs, il existe encore deux autres types qui sont la formation académique et professionnelle. Selon Jean Pierre Cuq: "La formation générale ou académique centrée sur les savoirs s'oppose à la formation professionnelle centrée sur les compétences et orientée sur la pratique d'un métier." (Cuq, 2003, p. 104)Nous déduisons que la formation professionnelle renvoie au métier exercé, dans ce contexte il s'agit bel et bien de l'enseignement des langues.

En France, en 1989 le rapport Bancel souligne qu'il faut professionnaliser les enseignants et définir les compétences que devrait maitriser un professionnel (Raynal, 1998, p. 403)

Comme le souligne Altet, désormais on ne forme plus au métier d'instituteur ou au professeur du second degré, mais on forme des professionnels de l'enseignement.

Nous reprenons le vocable utilisé par Huberman qui préfère dénommer l'enseignant en professionnel, voire un " clinicien de l'apprentissage " (Raynal, 1998, p. 404)

Selon Michel Dabène cité par j-P Cuq et I Gruca Les formation initiales et continue devraient comprendre:

-une formation disciplinaire,

-une formation sur la discipline ;

Une formation en didactique des disciplines ;

-une formation professionnelle au métier d'enseignant ;

-une formation à l'interdisciplinarité, en direction de la langue maternelle des élèves ." (Gruca, 2017, pp. 136-137).

\footnotetext{
${ }^{3}$ École normale supérieure de Bouzaréah désormais ENS -B.
} 
Nous pouvons conclure que les formations doivent englober pour former les professionnels du métier: une connaissance des savoirs disciplinaires, sur la didactique de la discipline, sur le métier de l'enseignant et une ouverture sur les disciplines, voire ouverture sur les langues et la langue première L1.

\section{3-La formation des formateurs à 1'ENS de Bouzaréah}

En Algérie, l'institution qui est chargée de la formation formateurs en langues est bel et bien L'Ecole Normale supérieure 1'ENS qui se répartir dans le territoire national: ENS de Laghouat, de sétif, Ouargla et de Bouzaréah. L'article 20du décret excécutif n $166176 \mathrm{du}$ 14 juin 201624 fixant le statut-type de l'école normale supérieure stipule que l'une des missions attribuées à cette institution est: "d'assurer la formation au métier d'enseignant au profit du secteur de l'Education nationale. " ( EN)

La formation à l'ENS comprend six disciplines en sciences humaines: 1'histoire -géographie, les lettres arabes, la philosophie ,l'amazight, la langue français et enfin la langue anglaise.

Il est à souligner que la formation des PES est dispensée à l'ENS pour tous les départements excepté le département d'Amazight. Pour le moyen, deux départements ne disposent pas de ce profil: Amazight et philosophie. Enfin pour le profil PEP, trois départements seulement l'assurent: l'arabe, le français et l'amazight.

A la fin de cette formation (fin de cycle) les étudiants- stagiaires sont orientés aux établissements de l'Éducation Nationale pour effectuer leur stage pratique sur deux temps: observation et intervention. A la fin des évaluations, l'étudiant aura un diplôme destiné à l'enseignant avec un contrat de travail. Nous allons dans ce qui suit aborder la place de la didactique du plurilinguisme dans les programmes de formation des formateurs pour les deux départements de langues ( français et anglais).

\section{4-Description des programmes de formation au plurilinguisme dans les départements de langues}

L'examen des programmes de formation dans les deux départements (anglais et français) nous révèle que la dimension plurilingue est étudiée durant la troisième année universitaire (3ème PEM, 3ème PES ) pour les deux départements. Cette notion n'est étudiée que d'un point de vue linguistique dans les modules intitulés: sociolinguistique et linguistique contrastive.

Les thématiques abordées renvoient dans leur majorité à:

-au bilinguisme

-Bilingualism and Multigualism

-le plurilinguisme, le contact des langues, attitudes et représentations, politiques éducatives.

Pour ce qui est du module de linguistique contrastive, les deus départementx l'intègrent en troisième année ( 3 ème) pour le département de français (Pem et PES ) et en quatrième année pour le département d'anglais. Les principaux points abordés dans ce module sont:

-l'analyse des erreurs

- les erreurs interlinguales ou les interférences - les erreurs interlinguales

4Cet article a été publié dans le Journal Officiel de la République Algérienne n^36 publié le 19 juin 2016. 
Il est à noter aussi que les modules de didactique préparant à la professionnalisation au métier d'enseignant sont étudiés à la dernière année de formation sans évoquer la didactique du plurilinguisme. Il s'agit pour la langue française de trois modules: didactique générale, didactique de la discipline et étude des programmes et manuels. Pour la langue anglaise: nous citons également ces trois modules Syllabus design -Applied linguistics Pedagogical trends.

\section{5-Méthode}

\section{5-1-1'entretien semi-directif}

Pour traiter cette thématique, nous avons eu recours à la méthode qualitative basée sur l'entretien semi-directif car nous estimons que les enquêtés s'expriment de manière fiable nous fournissant des données plus tangibles.

Cet entretien comprend neuf questions majoritairement ouvertes s'adressant aux enseignants de langues (anglais et français) et appartenant aux deux spécialités didactique et de sciences du langage.

L'objectif de cet entretien est de voir la place accordée au plurilinguisme dans la formation des futurs enseignants. Notre objectif est de s'interroger sur la place des approches plurielles dans les programmes de formation initiale.

Pour ce faire, nous avons interrogé huit enseignants universitaires permanents affiliés aux départements de langues (français et anglais). Le choix des deux départements s'explique par le fait que ces derniers sont chargés de former les futurs enseignants et où la compétence plurilingue est omniprésente ; il s'agit donc pour nous d'effectuer une étude comparative dans les deux départements. Nous avons rassemblé les questions de cet entretien et leurs items dans le tableau ci-dessous.

Tableau 1: Questions et items de l'entretien semi-directif

\begin{tabular}{|l|l|}
\hline \multicolumn{1}{|c|}{ Questions } & \multicolumn{1}{|c|}{ Items } \\
\hline $\begin{array}{l}\text { 1-Depuis quand enseignez-vous à } \\
\text { l'université ? }\end{array}$ & expérience à l'université \\
\hline $\begin{array}{l}\text { 2-que représente le plurilinguisme pour } \\
\text { vous? }\end{array}$ & maitrise du concept \\
\hline $\begin{array}{l}\text { 3-en tant qu'enseignant de langue, tolérez- } \\
\text { vous l'usage des langues en classe? }\end{array}$ & $\begin{array}{l}\text { savoir la place accordée à la compétence } \\
\text { plurilingue en classe }\end{array}$ \\
\hline $\begin{array}{l}\text { 4-si non, comment le trouvez-vous? } \\
\text { Bénéfique inadmissible excellent }\end{array}$ & $\begin{array}{l}\text { représentations des enseignants sur la place } \\
\text { de cette compétence }\end{array}$ \\
\hline $\begin{array}{l}\text { 4-avez-vous été formé au plurilinguisme ? } \\
\text { 5-comment abordez-vous la compétence } \\
\text { plurilingue dans vos cours ? }\end{array}$ & $\begin{array}{l}\text { savoir si les enseignants ont été formés sur c } \\
\text { concept, }\end{array}$ \\
\hline $\begin{array}{l}\text { 6-vos étudiants font -ils recours suivie pour cet enseignement } \\
\text { constamment à cette compétence ? }\end{array}$ & $\begin{array}{l}\text { savoir si les étudiants utilisent les } \\
\text { différentes langues }\end{array}$ \\
\hline $\begin{array}{l}\text { 7-est ce que l e programme de formation } \\
\text { comprend la compétence plurilingue? }\end{array}$ & $\begin{array}{l}\text { place de la compétence dans les formation } \\
\text { des formateurs }\end{array}$ \\
\hline
\end{tabular}




\begin{tabular}{|l|l|}
\hline 8- comment trouvez-vous ce programme ? & $\begin{array}{l}\text { Perception du programme dédiée à la } \\
\text { compétence plurilingue }\end{array}$ \\
\hline $\begin{array}{l}\text { 9-Quelles sont vos recommandations pour } \\
\text { améliorer cet enseignement? }\end{array}$ & $\begin{array}{l}\text { énumérer les recommandations - } \\
\text { propositions pour une bonne formation au } \\
\text { plurilinguisme. }\end{array}$ \\
\hline
\end{tabular}

\section{5-2-L'échantillon}

Les enseignants ayant participé à cet entretien sont en nombre de huit, à raison de deux enseignants de chaque spécialité citée supra. Nous avons choisi ces deux spécialités car à notre sens elles nous montrent effectivement la place de la pluralité linguistique proprement parler.

Nous présentons dans ce qui suit l'entretien semi-directif. Celui-ci comprend neuf questions ouvertes invitant les enseignants interrogés à nous fournir des informations relatives à:

-leur expérience dans l'enseignement universitaire ;

-la maitrise du concept du plurilinguisme ;

-leurs pratiques enseignantes et place accordée à la compétence plurilingue ;

-leur perception de cette compétence en classe de FLE ;

-savoir si les programmes de formation initiale incluent la pluralité et comment est -elle incluse ;

-savoir si la formation des formateurs préparent les enseignants futurs à faire face à cette compétence ;

-énumérer les recommandations et les propositions pour une formation de qualité en plurilinguisme.

\section{6-Résultats et discussion}

\section{6-1-Maitrise du concept de plurilinguisme}

L'analyse des résultats de l'entretien semi-directif montre que les enseignants interrogés ont une expérience entre deux à dix ans dans l'enseignement supérieur. Il a été démontré aussi que ces derniers possèdent une maitrise suffisante du concept. Nous avons retenu les prpos suivants:

" le plurilinguisme est le fait de maitriser plusieurs langues à l'oral et à l'écrit ;

-pouvoir passer d'une langue à une autre aisément ;

-penser-parler et écrire en plusieurs langues ;

-maitrise parfaite des langues en situation d'intersection. "

Nous remarquons que ces enseignants ont une maitrise minimale du concept qui selon Coste, D, D., Moore et G. Zarate: " On désignera par compétence plurilingue et pluriculturelle, la compétence à communiquer langagièrement et à interagir culturellement possédée par un acteur qui maîtrise, à des degrés divers, plusieurs langues, et a, à des degrés divers, l'expérience de plusieurs cultures," (Daniel COSTE, 2009, p. 11) Nous remarquons d'après cette définition qu'il ya imbrication entre langue et culture et que la compétence communicative est le point visé en privilégiant l'interaction. La maitrise des langues cependant n'est pas excellente, mais c'est à des degrés plus ou moins différents.

Pour une question qui a été posée aux enquêtés concernant leur propre formation au sujet du plurilinguisme, ceux-ci affirment à l'unanimité qu'ils ont été formés à propos de ce concept dans les modules de graduation( licence) juste pour ce qui est des modules des sciences du langage, à savoir la linguistique constrastive ou en sociolinguistique. Ceci s'applique aussi pour ce qui de la langue anglaise, dans les modules de linguistics et 
applied linguistics. Ceux- ci rajoutent qu'ils n'ont eu aucune formation proprement dite au sujet de l'introduction de la compétence plurilingue en classe de langue " nous n'avons pas étudié la didactique du plurilinguisme durant notre formation initiale. ". Les enseignants de didactique confirment à l'unanimité l'absence de contenus proposés dans la formation initiale des enseignants des trois cycles (primaire, moyen et secondaire) au sujet du plurilinguisme. Ils rajoutent: "la didatique n'est étudiée qu'en fin de formation, c'est-à-dire la dernière année et lors du stage que les stagiaires enseignants découvrent l'intérêt de cette dimension en classe. Nous abordons les modules:EPM5, didactique générale et didactique de la discipline et c'est fait de manière rapide ! Sans qu'il ait pour autant un renvoi à cette dimension plurilingue. "Ils rajoutent aussi qu'ils ne l'ont vu que durant leur formation en master et en doctorat: " c'est en master et doctorat que j'ai bien reconnu l'importance de la didactique du plurilinguisme en classe de classe. "En effet, les interrogés sont dans leur majorité des doctorat ( 5 doctorants ) et trois docteurs seulement. Nous pouvons rajouter à la fin que la compétence plurilingue est en parfaite association avec la compétence pluriculturelle et ce comme l'annoncent j-P , Robert; Evelyne Rosen: " ce faisant , puisqu'apprendre une langue c'est intégrer la culture de cette langue, le Cadre associe la notion de pluriculturalisme à celle de plurilinguisme et parle de compétence plurilingue et pluriculturelle. "( (j-P \& Rosen, 2010, p. 208)

\section{6-2-Perception de la compétence plurilingue par les formateurs}

La majorité des enseignants ont confirmé qu'ils ne tolèrent pas l'usage d'une langue autre que celle qui est utilisée pour enseigner la spécialité (anglais ou français).Ils trouvent que ces langues représentent un obstacle pour les interlocuteurs et utilisateurs de la langue " au lieu de connaitre d'autres mots nouveaux, ils pensent en d'autres langues et ça c'est inadmissible. " d'autres disent: "nous avons été formés ainsi et nous allons le transférer à nos étudiants. "D'autres enseignants affirment que: "la langue aide oui, je leur parle de l'importance de cette compétence, mais on n'a pas de contenus propres à ça. " " c'est dommage, il faut actualiser."

\section{7-Recommandations pour une prise en charge de la compétence plurilingue en formation initiale}

Les enseignants universitaires interrogés émettent les recommandations suivantes pour une meilleure prise en charge dans la formation des futurs enseignants:

"-augmenter le volume horaire alloué aux modules de didactique ;

-rajouter les modules de didactique professionnelle et de la didactique professionnelle ;

-former les futurs enseignants à ce qui se passe réellement en classe de langue ;

-proposer des cours modèles où la compétence plurilingue est insérée ;

-sensibiliser les enseignants universitaires à l'importance de cette dimension et préparer les futurs formés à l'accepter ;

-expliquer à ces formés les stratégies à adopter à son égard ;

-faire une articulation entre les modules des sciences de langage et ceux de la didactique ;

- proposer des situations réelles (vidéos de classes) aux futurs enseignants pour qu'ils reconnaissent l'importance de la compétence plurilingue dans l'enseignement des langues. "

\section{8-Conclusion}

Cette recherche a pour objectif de reconnaitre la place accordée à la compétence plurilingue dans les programmes de formation des formateurs à l'ENS de Bouzaréah et ce en prenant en compte les deux départements de langues (anglais et français).

5 EPM: étude des programmes et des manuels scolaires, est un module de didactique. 
Nous avons posé le postulat que les programmes n'accordent pas une place de taille à cette compétence qui demeure marginalisée, refusée voire inadmissible dans le cours de langue à l'université. Nous avons pu savoir que les programmes de formation initiale ne prennent pas compte de cette compétence. Ces programmes insèrent juste cette compétence dans les modules de linguistique et d'une manière théorique sans application ni renvoi au contexte de la classe. Nous avons aussi déduit que les enseignants de didactique ont souligné qu'aucun programme en graduation n'aborde cette thématique, et s'il y a lieu de l'évoquer c'est rapidement et à la demande des enseignants stagiaires. L'analyse des résultats de l'entretien semi-directif a révélé aussi qu'il ya une non actualisation des programmes de formation initiale aux besoins actuels de la communication et aux approches plurielles.

Nous pensons qu'il donc plus qu'urgent de revoir les programmes de formation des formateurs et de prendre en considération cette compétence si nécessaire dans le cadre de la classe et ce à la demande des enseignants de la didactique surtout.

Nous tenterons dans des travaux futurs à nous focaliser sur le contexte de la classe de langue pour voir le fonctionnement réel de cette compétence, nous envisageons d'effectuer des observations de classe dans les trois cycles d'enseignement (primaire, moyen et secondaire) afin d'examiner de près la présence de la pluralité linguistique en classe de langue. L'objectif étant de pouvoir proposer un dispositif didactique que l'on pourrait expérimenter en classe et ce à travers la conception d'un projet didactique plurilingue.

\section{Bibliographie:}

Causa, M. (2012). . Réflexions autour de la mise en place d'une éducation au(x) plurilinguisme(s) en formation initiale à l'enseignement des langues. DIversité REcherches et terrains, (2). https://doi.org/10.25965/dire.255

Cuq, J.-P. (2003). Dictionnaire de didactique du français langue étrangère et seconde . Paris: Cle international.

Cuq, J-p \&Gruca, I. (2017). Cours de didactique de français langue étéragère et seconde. Grenoble: PUG.

Coste, D, D., Moore et G. Zarate.(2009) .Compétence plurilingue et pluriculturelle. Strasbourg: Ed du Conseil de l'Europe.

Derivry-Plard, M. (2015). Paradigme plurilingue et pluriculturel: enseignants et didactique des langues, théories et pratiques. Université de la Sorbonne Nouvelle, Paris 3.: HDR, non publiée.

Robert, J-P \& Rosen, E. (2010). Dictionnaire du cecrdictionnaire pratique du CECR,Pour un CECR accessible à tous les professeurs de langues. Avec un glossaire des termes-clés en 13 langues. Paris: Editions OPHRYS.

Klet, E. (2017). Internationalisation, plurilinguisme et formation des enseignants de langues étrangères. Synergies Argentine (5), pp. 57-70.

Raynal,F\& Rieunier, A. (1998). Pédagogie; dictionnaire des concepts clés de pédagogie Apprentissage, formation, psychologie cognitive. Paris: ESF. 